\title{
ТЕХНИКО-ЭКОНОМИЧЕСКАЯ ОПТИМИЗАЦИЯ РЕАКТОРОВ, НАХОДЯЩИХСЯ В СЛОЖНОЙ ХИМИКО-ТЕХНОЛОГИЧЕСКОЙ СИСТЕМЕ
}

В последнее время большое значение в химической промышленности приобретает оптимизация химико-технологических схем с точки зрения экономической эффективности производства. Критерием оптимизации при этом обычно является обобщенный экономический показатель - приведенный доход или приведенные расходы ['].

В задаче оптимизации реакторов в системе могут быть два крайних подхода: оптимизируется каждый аппарат в отдельности или оптимизируется целая система. Оба подхода широко обсуждаются в литературе, но они имеют общеизвестные недостатки. В настоящее время наиболее приемлемым считается такой подход, когда процесс оптимизации схемы должен рассматриваться как процесс последовательных приближений, на первом этапе которого проводится оптимизация реакторов и других аппаратов схемы на основе тех или иных локальных критериев, а на втором этапе - оптимизация всей схемы [2].

Нами предлагается такой подход, когда критерий формируется таким образом, чтобы в нем отражалась вся информация о всей системе, уже известная до данного этапа оптимизации системы. Из методов оптимизации подробнее мы останавливаемся на использовании принципа максимума, позволяющего определить распределение параметров по длинє аппаратов. Однако приведенную ниже методику расчета целевой функции можно применять при любом методе оптимизации. Предлагаемая нами методиқа расчета целевой функции является общеиспользуемой. Здесь принимается во внимание непосредственно структура схемы, и, как нам представляется, эта методика эффективна не только при оптимизации схем заданной структуры, но и (даже особенно) при нахождении оптимальной структуры схемы.

Одной из основных проблем при технико-экономической оптимизации с применением принципа максимума является нахождение функционала, дающего критерий оптимизации. Нами выводится один из видов такого функционала, имеющий применение при обширном классе процессов.

Сущность нашего подхода можно рассматривать в двух, относительно самостоятельных, частях: образование и расчет целевой функции и оптимизация.

Образование и расчет целевой функци и. За критерий оптимизации принимаются приведенные расходы производства целой системы. Все аппараты в схеме делятся на две группы: оптимизируемые и неоптимизируемые на данном этапе. Неоптимизируемые блоки мы 
описываем с помощью операционных матриц. Для описания оптимизируемых блоков нами используются точные физико-химические математические модели, которые обычно являются системами обыкновенных или дифференциальных уравнений. Но результаты действия этих моделей на входные потоки можно также представить в виде «фиктивных» матриц, которые в конкретном случае являются операционными. Отличие их от других операционных матриц состоит в том, что элементы «фиктивных» матриц не постоянны, а меняются в зависимости от изменения (оптимизащии) параметров точных математических моделей. Теперь на основе всех операционных матриц схемы и при известной структуре схемы можно найти эквивалентную матрицу преобразования системы. На базе этой матрицы возможны определение всех потоков в схеме и расчет приведенных расходов производства системы.

Опти мизация. Ниже рассмотрим случай, когда оптимизируется лишь один реактор, так как применение данного метода в случае нескольких реакторов не представляет трудностей. Математическое описание процесса имеет вид:

$$
\frac{d x_{i}}{d \tau}=r_{i}(\bar{x}, \bar{u}) \quad(i=1,2, \ldots, m),
$$

где $\bar{x}=\left(x_{1}, x_{2}, \ldots, x_{m}\right)-$ состояние процесса и $\bar{u}=\left(u_{1}, u_{2}, \ldots, u_{s}\right) \in U-$ вектор управления, $U$ - допустимая область определения векторов управления. ции $\tau_{k}$

Пусть приведенные расходы $\Psi$ зависят от времени окончания реак-

$$
\Psi=\Psi\left(\tau_{k}\right) \quad\left(0 \leqslant \tau_{k} \leqslant \tau_{\mathrm{occM}}\right),
$$

где $\tau_{\text {осмм }}$ - граница рассматриваемого промежутка времени.

За значение $\Psi(0)=\Psi_{0}$, которое отвечает приведенным расходам при нулевой степени превращения реагентов, нужно принять фиктивное «большое» число, обусловленное ниженазванным требованием. Вектор управления следует определять так, чтобы приведенные расходы $\Psi\left(\tau_{k}\right)$ были минимальные. Значение $\tau_{k}$ можно фиксировать или же оставить свободным.

Задачу можно сформулировать как задачу минимизации функционала $I$, где

$$
I=\int_{0}^{\tau_{k}} \frac{\partial \Psi}{\partial \tau} d \tau \quad\left(0 \leqslant \tau_{k} \leqslant \tau_{\mathrm{occM}}\right)
$$

так как

$$
I=\Psi\left(\tau_{k}\right)-\Psi(0)=\Psi\left(\tau_{k}\right)-\text { const. }
$$

Принимаем, что функция

$$
I(\tau)=\int_{0}^{\tau} \frac{\partial \Psi}{\partial \tau} d \tau \quad\left(0 \leqslant \tau \leqslant \tau_{k}\right)
$$

монотонная. Это требование обычно выполняется, поскольку приведенные расходы (2) описываются как монотонно убывающая функция времени окончания реакции в промежутке $\left[0, \tau_{k}^{\prime}\right] \quad\left(0 \leqslant \tau_{k} \leqslant \tau_{k}^{\prime} \leqslant \tau_{\text {осмм }}\right)$, где $\tau_{k}^{\prime}$ - оптимальное время окончания реакции. Причем скорость этого убывания замедляется с приближением $\tau_{k}$ к оптимальному значению 
$\tau_{k}^{\prime}$. Можно доказать, что в таком случае задачу можно свести к задаче о быстродействии [3].

При нефиксированном значении $\tau_{k}$. задачу можно сформулировать следующим образом: найти оптимальную траекторию $\bar{u}_{\text {опт }}(\tau)$ и значение $\tau_{k}$ из условия

$$
H\left(\lambda_{0}, \bar{\lambda}(\tau), \bar{x}^{\prime}(\tau), \bar{u}_{\text {oIT }}(\tau)\right)=\max _{\bar{u} \in \mathcal{E}_{\tau \leqslant \tau_{k}}} H\left(\lambda_{0}, \bar{\lambda}(\tau), \bar{x}^{\prime}(\tau), \bar{u}(\tau)\right) \equiv 0,
$$

где функция $H$ имеет вид

$$
\begin{array}{r}
H\left(\lambda_{0}, \bar{\lambda}(\tau), \bar{x}^{\prime}(\tau), \bar{u}(\tau)\right)= \\
=\lambda_{0} \frac{\partial \Psi}{\partial \tau}+\sum_{k=1}^{m} \lambda_{k}(\tau) r_{h}\left(\bar{x}^{\prime}(\tau), \bar{u}(\tau)\right)+\lambda_{m+1}
\end{array}
$$

и вектор $\bar{x}^{\prime}=\left(x_{1}, x_{2}, \ldots, x_{m}, \tau\right)$.

В данном случае функция $\lambda_{0}$ равна неположительной константе $\lambda_{0}=$ const $\leqslant 0$. Поскольку функции $\lambda_{i}(\tau)$ при интегрировании описывающей их системы уравнений (8) находятся с точностью до произвольного постоянного множителя, можно считать $\lambda_{0}=-1$. Следовательно, функция $H$ (6) имеет вид

$$
H=--\frac{\partial \Psi}{\partial \tau}+\sum_{k=1}^{m} \lambda_{k}(\tau) r_{k}\left(\bar{x}^{\prime}(\tau), \bar{u}(\tau)\right)+\lambda_{m+1}
$$

Функции $\lambda_{k}(\tau)(k=1,2, \ldots, m+1)$ определяются из системы уравнений:

$$
\begin{aligned}
& \frac{d \lambda_{k}}{d \tau}=\frac{\partial^{2} \Psi}{\partial \tau \partial x_{k}}-\sum_{i=1}^{m} \lambda_{i}(\tau) \frac{\partial r_{i}\left(\bar{x}^{\prime}(\tau), \bar{u}_{\text {oпт }}(\tau)\right)}{\partial x_{k}} \\
& \frac{d \lambda_{m+1}}{d \tau}=\frac{\partial^{2} \Psi}{\partial \tau^{2}}
\end{aligned}
$$

а функции $x_{k}(\tau)(k=1,2, \ldots, m)$ - из системы уравнений $(1)$.

При фиксированном значении времени окончания реакции постановка задачи меняется по известным правилам. Вопрос о граничных условиях рассматривается также согласно общепринятой методике [3, 4].

В качестве примера рассмотрим применение вышеописанной методики при оптимизации реактора в основной стадии производства синтетических душистых веществ.

Структурная схема основной стадии производства синтетических душистых веществ.

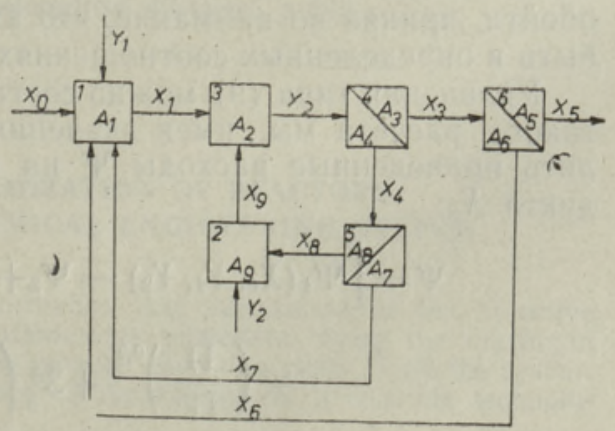


На приведенной схеме (рисунок) блоки обозначены ящиками и пронумерованы: $1, \ldots, 6$, потоки обозначены стрелками. В ящиках и над стрелками даны обозначения соответствующих операционных матриц и векторов потоков. Система состоит из следующих процессов: двух процессов смещения -1 (матрица $\left.A_{1}\right)$ и $2\left(A_{9}\right)$; трех процессов разделения на два потока $-4\left(A_{3}\right.$ и $\left.A_{4}\right), 6\left(A_{5}\right.$ и $\left.A_{6}\right)$ и $7\left(A_{7}\right.$ и $\left.A_{8}\right) ;$ одного процесса кимического превращения $5\left(A_{2}\right)$.

При образовании эквивалентной матрицы преобразования системы можно учесть своеобразие рассматриваемой системы. Зная, что входные потоки $Y_{1}$ и $Y_{2}$ зависят от промежуточных потоков системы, можно найти матрицы $B_{1}$ и $B_{2}$ так, что

$$
\begin{aligned}
& Y_{1}=B_{1}\left(X_{0}+X_{6}+X_{7}\right), \\
& Y_{2}=B_{2} X_{1}-X_{8} .
\end{aligned}
$$

Можем ограничиться рассмотрением только таких потоков, в которых имеются вещества, рециркулирующие в системе. Операционные матрицы процессов смещения являются единичными, и, следовательно, при умножении матриц ими можно пренебречь.

При расчете эквивалентной матрицы преобразования можно использовать метод свертки на основе информации структурного анализа ["]. Эквивалентная матрица преобразования данной системы следующая:

$$
X_{5}=A_{5} \dot{A}_{3} A_{2}\left(I+B_{2}\right)\left[I-\left(I+B_{1}\right)\left(A_{7} A_{4}+A_{6} A_{3}\right) A_{2}\left(I+B_{2}\right)\right]^{-1}\left(I+B_{1}\right) X_{0},
$$

где $I$ - единичная матрица.

Блок 3 является оптимизируемым, следовательно, матрица $A_{2}-$ «фиктивной». Обычно «фиктивную» матрицу $A$ можно представить в виде диагональной $\left(a_{i j}=0, i \neq j\right)$. Пусть математическая модель устанавливает соответствие между входным потоком $X$ и выходным потоком $Y(X \stackrel{\boldsymbol{A}}{\rightarrow} Y)$, тогда за диагональные элементы матрицы $A$ принимаем

$$
a_{i i}=\frac{y_{i}}{x_{i}} .
$$

Если $x_{i}=0$ и $y_{i}=0$, то $a_{i i}=0$, а если $x_{i}=0$ и $y_{i} \neq 0$, то нельзя использовать «чисто» диатональную матришу, в строке $i$, где $x_{i}=0$, нужно некоторое $a_{i j} \neq 0$, где $j \neq i$, так что $Y=A X$.

Входной поток $X_{1}$ в оптимизируемый блок 3 зависит от «фиктивной» матрицы $A_{2}$ :

$$
X_{1}=\left[I-\left(I+B_{1}\right)\left(A_{7} A_{4}+A_{6} A_{3}\right) A_{2}\left(I+B_{2}\right)\right]^{-1}\left(I+B_{1}\right) X_{0} .
$$

Следовательно, возникает итеративный процесс. Но обычно это можно обойти, приняв во внимание, что входящие в реактор вещества должны быть в определенных соотношениях.

Уравнения типа (9) можно составить и для остальных потоков. После такого расчета мы, имея значения всех потоков схемы, можем вычислить приведенные расходы $\Psi$ на производство единицы целевого продукта $X_{5}$ :

$$
\begin{gathered}
\Psi=\left[\Psi_{1}\left(X_{0}, Y_{1}, Y_{2}\right)-\Psi_{2}+3_{\frac{7}{0}} \frac{\Pi}{\Pi^{0}}+\left(3_{\mathrm{a}}^{0}+3_{\mathrm{p}}^{0}+\mathrm{EK}_{\mathrm{T}}^{0}\right) \times\right. \\
\left.\times\left(\frac{\Pi}{\Pi^{0}}\right)^{0,7}+3_{3}^{0}\left(\frac{\Pi}{\Pi^{0}}\right)^{0,1}+3_{\mathrm{H}}^{0}\right] / X_{5},
\end{gathered}
$$


где $\Psi_{1}$ - стоимость сырья в зависимости от входящих в систему потоков и цен сырьевых компонентов, $\Psi_{2}$ - стоимость побочных продуктов и реализуемых отходов (в рассматриваемом случае $\left.\Psi_{2}=0\right) . \quad 3_{a}^{0}, 3_{\mathrm{a}}^{0}, 3_{\mathrm{p}}^{0}$, $3_{3}^{0}, 3_{\text {н }}^{0}, \mathrm{~K}_{\mathrm{T}}^{0}, \Pi^{0}$, - соответственно, энергетические расходы, амортизационные отчисления, затраты на ремонт, заработная плата и начисления, цеховые и общезаводские расходы, производственные фонды и объем главного потока, - все показатели определены по базовому варианту производства на опытно-производственной установке, E - нормативный коэффициент эффективности капиталовложений, П - текущее значение объема главного потока (в данном случае потока $X_{2}$ ). С помощью уравнения (10) можно рассчитать целевую функцию и использовать ее в процедурах оптимизации, например, в случае примене'ния принципа максимума, согласно вышеприведенным уравнениям $(1-8)$.

Нами найдены оптимальные температурные профили реакционной смеси и теплоносителя, а также оптимальная степень превращения для трубчатого реактора гомогенного жидкофазного катализа (блок 3), являющегося основным звеном данного технологическото процесса.

\section{ЛИТ Е РА Т У Р А}

1. О с т ров ски й Г. М., В олин Ю. М. Моделирование сложных химико-технологических схем. М., 1975.

2. Ост ров ски й Г. М., С ли н ько Н. Г. Оптимизация химических реакторов и процессов. - Теор. основы хим. технол., 1975, т. 9, № 6, с. 853-862.

3. Болтян ски й В. Г. Математические методы оптимального управления. М., 1969.

4. Боя ринов А. И., Кафаров В. В. Методы оптимизации в химической технологии. М., 1971.

5. Кирьянен И., Р а яло Г. Программа структурного анализа сложных химикотехнологическнх схем. Таллин, препринт К-3, Отд. хим., геол. и биол. н. АН ЭССР, 1977.
Ннститут химии
Академии наук Эстонской ССР
Поступила в редакцию 29/XII 1977

\section{KIRJANEN, G. RAJALO}

\section{KEERUKASSE KEEMILIS-TEHNOLOOGILISSE SUSTEEMI KUULUVATE REAKTORITE TEHNILIS-MAJANDUSLIK OPTIMEERIMINE}

Artiklis on esitatud metoodika, mis käsitleb sihifunktsiooni moodustamist, arvutamist ning rakendamist reaktori optimeerimisel maksimumprintsibi alusel. Optimeerimiskriteeriumiks on kogu süsteemi taandatud tootmiskulud toodanguühiku kohta. On esitatud näide meetodi rakendamisest konkreetse tehnoloogilise süsteemi puhul.

\section{KIRJANEN, G. RAJALO}

\section{TECHNO-ECONOMICAL OPTIMIZATION OF REACTORS BELONGING TO A COMPLEX CHEMICAL ENGINEERING SYSTEM}

An optimization method, consisting of the formation and calculation of the objective function and its application to the reactor optimization procedure, using the maximum principle, is described. As optimum criterion, the reduced operating costs of all the system are used. An example regarding the application of the method to a concrete technological system is presented. 